\title{
Physical Therapy for Esophageal Cancer Patient With Long Thoracic Neuropathy After Esophagectomy: A Case Report
}

\author{
Junghwa Do¹, PhD, DPT, One-bin Lim², PhD, PT, Ja-young Kim, MD, Jae Yong Jeon ${ }^{1}, \mathrm{PhD}$, MD, Young-ki Cho', \\ PhD, PT
}

${ }^{1}$ Department of Rehabilitation Medicine, Asan Medical Center, University of Ulsan College of Medicine, Seoul, ${ }^{2}$ Department of Physical Therapy, College of Health Science, Yonsei University, Wonju, Korea

\author{
Article Info \\ Received July 3, 2020 \\ Revised July 22, 2020 \\ Accepted July 31, 2020 \\ Corresponding Author \\ Jae Yong Jeon \\ E-mail: jyjeon71@gmail.com \\ https://orcid.org/0000-0003-1534-7931
}

\section{Key Words}

Esophageal neoplasms

Exercise

Pain

Quality of life
Esophageal cancer is a representative cancer that occur physical deterioration but, physical problems after surgery were not well reported. The purpose of this study is to report on the long thoracic neuropathy after surgery, and to identify the symptoms and effects of physical therapy after esophageal cancer surgery. This is a case of a 61-year-old man who showed winging of the scapula with long thoracic nerve injury on the results of electromyography after an esophageal cancer surgery. Physical therapy programs were implemented 8 sessions during hospitalization. The quality of life, fatigue, shoulder range of motion (ROM), numeric rating scale (NRS), 6-minute walk test, and 30-second chair stand test were assessed. The quality of life, fatigue, shoulder ROM, NRS (pain), 6-minute walk test, and 30-second chair stand test were improved. However, the esophageal-specific symptom was not different after physical therapy program. As esophageal cancer suffers from physical difficulties after surgery, physical therapy programs are thought to be helpful.

\section{INTRODUCTION}

Esophageal cancer is defined as tumors along the lining of the muscular tube that connects the throat to the stomach [1]. It often occurs in men aged 60-70 years and accounts for $2.4 \%$ of all cancer deaths in South Korea in 2016 [2]. Despite the advances in cancer treatment, its prognosis remains poor. However, compared to surgery alone, patients who are eligible for neoadjuvant chemotherapy and radiation therapy achieved approximately $47 \%$ in a 5 -year survival rate [3].

Esophagectomy is the standard surgery for esophageal cancer, but is a well-known invasive procedure. Therefore, it affects postoperative complications, such as pulmonary complications, anastomotic leaks, and sepsis [4]. Postoperatively, patients with esophageal cancer may experience not only high-risk postoperative pulmonary complication but also pain, fatigue, eating difficulties, diarrhea, nausea, and impaired quality of life (QoL) outcomes [5,6]. Furthermore, sarcopenia, weight loss, and physical deterioration are also well document- ed as physical side effects. One study reported that the 6-minute walk test and knee extensor muscle test decreased within 3 weeks after esophagectomy [7]. Decreased cardiorespiratory ability and muscle dysfunction affected the survival [8].

Several studies showed that physical exercise is beneficial in the aspect of QoL after completing the treatment [9-11]. Moreover, the 2-week rehabilitation program during hospital stay after esophagectomy reported an effective strategy in decreasing pulmonary complication. The incidence of pulmonary complications was $8.8 \%$, which is lower than the previous studies. In this study, rehabilitation program was consisted of positioning, stretching of respiratory muscles and thoracic cage, deep diaphragmatic breathing, coughing and huffing, and early mobilization [12]. This study, postoperative rehabilitation focuses on reducing lung complications. However, there was no approach in identifying and treating musculoskeletal. Also, there is still a lack of research to show the beneficial effect of physical therapy program, and no study has included the description status of the skeletal muscle immediately after 
an esophageal cancer surgery Above all, there were no clinical research of neuropathy symptoms after esophagectomy.

This case report aimed to identify the symptoms immediately after an esophageal cancer surgery and the effects of physical therapy program on physical function, fatigue, pain, and QoL. Also, we would like to share the uncommon symptom of winging scapula which occurred on a surgical site after an esophageal cancer surgery.

\section{CASE REPORT}

\section{Subjects}

The patient is a 60-year-old man diagnosed with malignant esophageal neoplasm on March 23, 2018, with chest pain and mild dysphagia. His height was $163.2 \mathrm{~cm}$ and weight was 61.1 $\mathrm{kg}$, with a body mass index of $22.94 \mathrm{~kg} / \mathrm{m}^{2}$, with no metastasis or comorbidity. He underwent concurrent chemoradiation therapy preoperatively. He was admitted on June 16, 2018, and underwent a Vinci Ivor Lewis operation on June 19, 2018, which showed that proximal, distal, and radial resection margins were not involved and no metastasis in 28 lymph nodes. Therefore, he was diagnosed with stage cT2N1M0 esophageal squamous cell carcinoma.

The patient was examined in the physical therapy room on June 20, 2018, for postoperative lung care. There were no other treatment programs other than physical therapy. $\mathrm{He}$ complained of pain on the chest tube and medial border sites in the right scapula. He also complained that he could not lie down on the bed below $60^{\circ}$. Thus, the changed sleep position and pain resulted in insomnia, which he reported as the most terrible symptom among other things. He more especially complained of shoulder pain and weakness than other patients who underwent esophagectomy. On physical examination, severe winging scapula in the right shoulder was noted (Figure 1). It was a positive in wall push up test. And, strength of serratus anterior muscle was poor in manual muscle test. On the results of electromyography (EMG) finding, abnormal spontaneous potentials were observed in the right serratus anterior muscles during rest, and polyphase unit activity potential of long duration was observed in the right serratus anterior muscles during moderate contraction. He was diagnosed with axonotmesis in the long thoracic nerve on EMG examination. This study was approved by the Asan Medical Center Institutional Review Board (approval No. 2018-1349).

\section{Physical Therapy Program}

On his initial visit to physical therapy, the patient's chief complaint was shoulder pain and insomnia, but he also had a difficulty of expectoration due to pain in the surgical area. In addition, the 6-minute walk test showed decreased cardiopulmonary function due to decreased physical activity during pre-cancer radiation treatment. Therefore, physical therapy program consisted of breathing, chest expansion, soft massage, stretching, strengthening, and aerobic exercises. Physical therapy was performed for 60 minutes every day except weekends, and a total of eight sessions of physical therapy were received until discharge the hospital on July 1, 2018.

On the first day, the patient complained of severe pain on the rhomboid, i.e., level 5 on numeric rating scale (NRS) even during rest. Therefore, the physical therapist performed tolerable chest expansion and deep breathing exercise, right shoulder range of motion exercise, and diaphragm strengthening exercise in half sitting position. Incentive spirometry exercises using a coaching device (Coach II, Kendal; Tyco Healthcare, Mansfield, MA, USA) were recommended 10 times every hour as possible. For sputum excretion, coughing and huffing exercises were performed using tough techniques. Since the next day, biceps curl, shoulder flexion, and horizontal abduction using elastic band (Hygenic Corp., Akron, OH, USA) and squatting exercise were performed to strengthen the upper and lower parts of the body. The patient was informed on his Borg rating of perceived exertion scale and exercised at a "somewhat hard" intensity of 11-14, regarded as an exercise intensity level of $50 \%-70 \%$ of one ROM for resistance training for $1-3$ sets of 10 repetitions. Physical therapist monitored the $\mathrm{SpO}_{2}$ and

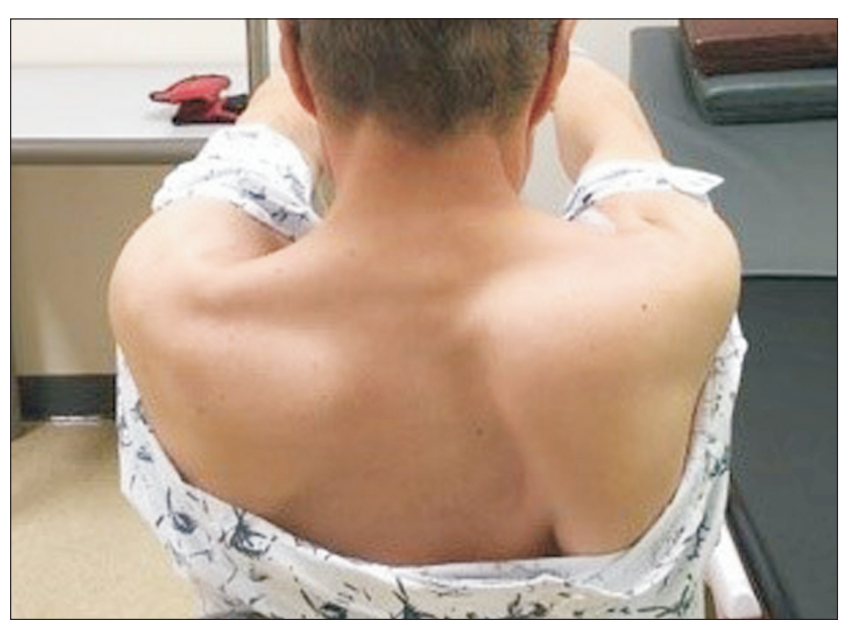

Figure 1. Winging scapula after esophagogectomy. 
heart rate during exercise. To exercise winging scapula, Serratus anterior muscle strengthening exercise was performed progressively from no weight to resistance exercise. In a standing position, shoulder elevation exercise was performed from $90^{\circ}$ to $120^{\circ}$ against the wall and using a hand weight in the plane of the scapula. He was standing with back towards the wall, feet shoulder-width apart, and grasping elastic band handles with both hands. Starting with elbows flexed at $90^{\circ}$ and shoulders abducted at $60^{\circ}$ and internally rotated at $45^{\circ}$, he then performed a hugging action by pressing the handles away from the body, forming an imaginary arc with his hands. Once the hands came into contact with each other, he slowly returned to the starting position following the same arc. And, stretching exercise to pectoralis minor, rhomboid and levator scapula was performed to prevent shortening. Additionally, a 5-minute soft massage by using manual pressure release was performed on rhomboid muscle due to severe pain on the rhomboid and levator scapula in the right scapular. Aerobic exercise was performed from 20 minutes at $50 \%$ of the predicted maximum heart rate to 30 minutes at 60\% progressively. Patients performed aerobic exercise using cycle (Ergoline 200k; Ergoline GmbH, Bitz, Germany) or SCI fit. Briefly, stationary cycle monitoring the target heart rate (HR: 90-108) was used with constant mode. Constant resistance was used from $20 \mathrm{~W}$ to $30 \mathrm{~W}$. The intensity of aerobic and strengthening exercises was referenced from the guidelines for sedentary people, provided by the American College of Sports Medicine. For cooling down, active range of motion exercise (AROM) on the neck and shoulder and chest expansion with deep breathing and stretching of the lower extremities were performed Stretching exercise to pectoral muscle was performed to prevent of $\mathrm{ky}-$ photic posture due to surgical pain and sleeping position.

\section{Assessments}

The European Organization for Research and Treatment of Cancer Core Quality of Life Questionnaire (EORTC QLQ-C30), EORTC Esophagus Questionnaire (EORTC QLQ-ES18), Functional Assessment of Chronic Illness Therapy-Fatigue (FACITfatigue), shoulder AROM, NRS, 6-minute walk test, and 30-second chair stand test were evaluated at the baseline and after the intervention.

\section{1) The 30-second chair stand test}

The 30-second chair stand test is performed by checking the chair stands for 30 seconds to assess the strength of the lower extremities. It has a moderately high test-retest reliability (intraclass correlation coefficient $=0.89)$ and moderate construct validity, as demonstrated by a correlation with the leg press $(r=$ $0.77)[13]$.

\section{2) The 6-minute walk test}

The 6-minute walk test was performed in a 30-m hallway at his own pace [14]. The physical therapist encouraged him "you' re doing well" and check the oxygen saturation at pre- and post-evaluation. Moreover, dyspnea was measured using the modified Borg dyspnea scale [15].

\section{3) QoL}

The Korean version of European Organization for Research and Treatment of Cancer Core Quality of Life Questionnaire; version 3 (EORTC QLQ-C30) was used to assess the QoL. This questionnaire is widely used for various cancer and has been validated [16]. The Korean version of Quality of Life Questionnaire-Esophageal 18 (QLQ-OES18) was used to evaluate esophageal symptom [17], which were highly reliable and validated as esophageal cancer-specific [18]. In this questionnaire, the higher score in function means the better physical function, and the higher the symptom means the more the symptoms.

\section{4) Fatigue}

The Functional Assessment of Chronic Illness Therapy-fatigue (FACIT-fatigue) was used to assess fatigue, which consists of thirteen questionnaires. This is validated for chronic disease, and higher score means less fatigue symptoms [19].

\section{5) AROM}

Each shoulder AROM such as flexion and abduction were measured while sitting. The average value of three trials was recorded according to [20].

\section{6) Pain}

The NRS was used to measure pain, with 0 indicating no pain and 10 indicating severe pain [21].

\section{Results}

Compared to baseline evaluation, at post treatment evaluation, the quality of life, physical function, role function, emo- 
tional function and cancer related symptom such as fatigue and insomnia (EORTC QLQ-C30). In aspect of esophageal specific symptom was not different except to pain (EORTC ESO18) (Table 1). Fatigue decreased from 15 points to 12 points in FACIT-fatigue. In addition, the pain score by NRS was reduced from 6 to 3. In the chair sit to stand test, he was able to do 10 times in the initial evaluation and 14 in the last evaluation. The 6-minute walk test also improved from 360 meters to 400 meters. The range of motion of the shoulder was limited in flexion and abduction at the baseline evaluation, but the shoulder $\mathrm{ROM}$ was restored to normal range after treatment (Table 2).

\section{DISCUSSION}

This case report is the first to describe a physical therapy program implemented immediately after the surgery and long thoracic neuropathy that occurred. The patient's compliance on the exercise program was very good. He showed severe

Table 1. Comparison of values obtained for EORTC QLQ-C30, EORTCOES18 between the baseline and after the physical therapy program

\begin{tabular}{|c|c|c|}
\hline \multirow{2}{*}{ Assessment } & \multicolumn{2}{|c|}{ Case } \\
\hline & Pre & Post \\
\hline \multicolumn{3}{|l|}{ EORTC QLQ-C30 } \\
\hline $\begin{array}{l}\text { Global health status/QoL } \\
\text { Functional scales }\end{array}$ & 50 & 58.3 \\
\hline Physical functioning & 46.6 & 53.3 \\
\hline Role functioning & 33.3 & 50 \\
\hline Emotional functioning & 75 & 83.3 \\
\hline Cognitive functioning & 100 & 100 \\
\hline Social functioning & 100 & 100 \\
\hline \multicolumn{3}{|l|}{ Symptom scales } \\
\hline Fatigue & 33.3 & 44.4 \\
\hline Insomnia & 100 & 33.3 \\
\hline Nausea and vomiting & 33.3 & 33.3 \\
\hline Pain & 66.6 & 50 \\
\hline Dyspnea & 100 & 33.3 \\
\hline \multicolumn{3}{|l|}{ EORTC QLQ-OES18 } \\
\hline Dysphagia & 55.5 & 55.5 \\
\hline Eating trouble & 66.6 & 66.6 \\
\hline Reflex & 66.6 & 66.6 \\
\hline Pain & 41.6 & 8.3 \\
\hline Dry mouth & 66.6 & 66.6 \\
\hline Taste trouble & 16.6 & 16.6 \\
\hline Coughing trouble & 26.6 & 26.6 \\
\hline Speaking trouble & 26.6 & 26.6 \\
\hline Trouble swallowing saliva & 66.6 & 66.6 \\
\hline Choking & 66.6 & 66.6 \\
\hline
\end{tabular}

EORTC QLQ-C30, The European Organization for Research and Treatment of Cancer Core Quality of Life Questionnaire; version 3; EORTC QLQ-OES18, The European Organization for Research and Treatment of Cancer Quality of Life Questionnaire-Esophageal 18. winging in the right scapula, mild dyspnea, and pain on the medial border of the scapula during the hospital stay. In addition, he also complained of severe insomnia due to posture changes during sleep.

Physical therapy programs after an esophageal cancer surgery are not widely implemented and generally, the referral from chest surgery part to rehabilitation medicine department is not clear. Therefore, physical problems after an esophageal cancer surgery were not well reported.

After surgery, occurrence of wining scapula ruled out of long thoracic nerve neuropathy. One case study also reported in case of an apical pulmonary tumor caused the long thoracic nerve palsy [22]. However, there have been no reports of long thoracic nerve damage after esophageal cancer surgery. Posterolateral thoracotomies are known to have the complication of long thoracic nerve palsies. Esophagectomy procedures include bilateral opening of the plural cavities; chest drain is placed on both sides to re-expand the lungs. Nerve damage might occur in this process [22]. The long thoracic nerve characteristically arises from 5 th to 7 th cervical nerve root [23]. The serratus anterior muscle weakness is innervated by the long thoracic nerve, which is important in controlling and maintaining normal scapulohumeral rhythm [24]. During the shoulder flexion, the serratus anterior muscle assists in the upward rotation and keeps the scapula to the thorax [25,26]. Therefore, we focused on exercises strengthening the serratus anterior. Shoulder elevation exercise was performed from $90^{\circ}$ to $120^{\circ}$ against the wall and using a hand weight in the plane of the scapula [27].

This patient had an improved QoL, better shoulder range of motion, and decreased pain and insomnia after the physical therapy program. This is consistent with the fact that physical

Table 2. Comparison of values obtained for FACIT-fatigue, NRS, and the 30-second chair stand test between the baseline and after the physical therapy program

\begin{tabular}{lrr}
\hline & \multicolumn{2}{c}{ Case } \\
\cline { 2 - 3 } & Pre & Post \\
\hline FACIT-fatigue (socres) & 15 & 12 \\
NRS (scores) & 6 & 3 \\
The 30-second chair stand test (times) & 10 & 14 \\
6-minute walk test (m) & 360 & 400 \\
Shoulder AROM (degrees) & & \\
$\quad$ Flexion & 125 & 180 \\
$\quad$ Abduction & 110 & 180 \\
\hline
\end{tabular}

FACIT-fatigue, Functional Assessment of Chronic Illness Therapy-Fatigue; NRS, numeric rating scale; AROM, active range of motion exercise. 
exercise and self-education program can benefit the postoperative QoL in patients with esophageal cancer [28]. He complained of severe insomnia. On the second day, he complained of severe insomnia. He reported that he slept just 1-hour because of uncomfortable postoperative position. Insomnia is a well-known significant problem in cancer patients [29]. Its prevalence in all cancer consists of 30-73\% of patients [30].

One study reported that patients diagnosed with esophageal cancer and healthy people in Europe had similar long-term health-related QoL, despite of persistent reflux and trouble eating [31]. Another study revealed that the physical and role functioning domains of QoL are lower in esophageal cancer compared to the control [32]. Comparisons between the preoperative and postoperative function showed significant decreases in 6-minute walk test, physical evaluation of SF-36, and improved hand grip and knee extensor muscle strength [12]. However, throughout the inspiration muscle training, coughing was not improved in the esophageal cancer symptom, it is thought to be the problem of the reflux caused by the swallowing. Multidisciplinary rehabilitation is recommended including oral hygiene, pulmonary rehabilitation, physical fitness, swallowing evaluation and rehabilitation, nutritional support, and pain management. Therefore, multimodal rehabilitative is required [33]. However, physical therapy program is not provided for esophageal cancer in most hospitals in South Korea. Although morbidity associated with esophageal cancer is high, patients had improved physical function and QoL, and various severe symptoms required more attention in rehabilitation medicine. Therefore, a physical therapy program during hospitalization are thought to be helpful in significantly reduced muscle strength and reduced lung function, even if neuropathy is not observed, such as patients in this case.

The limitations of this study were performed one case without control and short-term period for physical therapy. We could not discern the natural course of restore after surgery. Large-sized and long-term follow-up should be conducted to clarify this study's finding.

The physical therapy program had a positive effect on quality of life and fatigue in this patient. As esophageal cancer suffers from physical difficulties after surgery, physical therapy programs are thought to be helpful, and more active participation in rehabilitation programs is needed.

\section{AKNOWLEDGEMENTS}

This study was supported by a grant (2020-0596) from the Asan Institute for Life Sciences, Asan Medical Center, Seoul, Korea.

\section{CONFLICTS OF INTEREST}

No potential conflict of interest relevant to this article was reported.

\section{AUTHOR CONTRIBUTION}

Conceptualization: JD. Data curation: JD. Formal analysis: JD. Investigation: JD. Methodology: JYJ, YC. Resources: JK. Supervision: JYJ. Writing - original draft: JD. Writing - review \& editing: OL.

\section{ORCID}

Junghwa Do, https://orcid.org/0000-0002-9089-2086

One-bin Lim, https://orcid.org/0000-0002-5281-3463

Ja-young Kim, https://orcid.org/0000-0002-5010-8090

Young-ki Cho, https://orcid.org/0000-0003-2724-3128

\section{REFERENCES}

1. American Cancer Society. Esophagus cancer. American Cancer Society [Internet]. Atlanta: 2020 Mar [cited 2020 May 18]. Available from: http://www.cancer.org/Cancer/EsophagusCancer/OverviewGuide/index.

2. Korea Central Cancer Registry, National Cancer Center. Annual report on the causes of death statistics in Korea in 2016. Sejong: Ministry of Health and Welfare; 2018 Dec. Report No.: 11-1352000-000145-10. 13-5 p.

3. Sjoquist KM, Burmeister BH, Smithers BM, Zalcberg JR, Simes RJ, Barbour A, et al.; Australasian Gastro-Intestinal Trials Group. Survival after neoadjuvant chemotherapy or chemoradiotherapy for resectable oesophageal carcinoma: an updated meta-analysis. Lancet Oncol 2011;12(7):681-92.

4. Takeuchi H, Miyata H, Gotoh M, Kitagawa Y, Baba H, Kimura W, et al. A risk model for esophagectomy using data of 5354 patients included in a Japanese nationwide web-based database. Ann Surg 2014;260(2):259-66. 
5. Anandavadivelan P, Lagergren P. Cachexia in patients with oesophageal cancer. Nat Rev Clin Oncol 2016;13(3):185-98.

6. Djärv T, Lagergren J, Blazeby JM, Lagergren P. Long-term health-related quality of life following surgery for oesophageal cancer. Br J Surg 2008;95(9):1121-6.

7. Tatematsu N, Park M, Tanaka E, Sakai Y, Tsuboyama T. Association between physical activity and postoperative complications after esophagectomy for cancer: a prospective observational study. Asian Pac J Cancer Prev 2013;14(1):47-51.

8. Jack S, West MA, Raw D, Marwood S, Ambler G, Cope TM, et al. The effect of neoadjuvant chemotherapy on physical fitness and survival in patients undergoing oesophagogastric cancer surgery. Eur J Surg Oncol 2014;40(10):1313-20.

9. Gerritsen JK, Vincent AJ. Exercise improves quality of life in patients with cancer: a systematic review and meta-analysis of randomised controlled trials. Br J Sports Med 2016;50(13): 796-803.

10. Mishra SI, Scherer RW, Geigle PM, Berlanstein DR, Topaloglu O, Gotay CC, et al. Exercise interventions on health-related quality of life for cancer survivors. Cochrane Database Syst Rev 2012;(8):CD007566.

11. Fong DY, Ho JW, Hui BP, Lee AM, Macfarlane DJ, Leung SS, et al. Physical activity for cancer survivors: meta-analysis of randomised controlled trials. BMJ 2012;344:e70.

12. Inoue T, Ito S, Ando M, Nagaya M, Aso H, Mizuno Y, et al. Changes in exercise capacity, muscle strength, and healthrelated quality of life in esophageal cancer patients undergoing esophagectomy. BMC Sports Sci Med Rehabil 2016;8:34.

13. Jones CJ, Rikli RE, Beam WC. A 30-s chair-stand test as a measure of lower body strength in community-residing older adults. Res Q Exerc Sport 1999;70(2):113-9.

14. Guyatt GH, Thompson PJ, Berman LB, Sullivan MJ, Townsend $\mathrm{M}$, Jones NL, et al. How should we measure function in patients with chronic heart and lung disease? J Chronic Dis 1985;38(6):517-24.

15. Wilson RC, Jones PW. A comparison of the visual analogue scale and modified Borg scale for the measurement of dyspnoea during exercise. Clin Sci (Lond) 1989;76(3):277-82.

16. Yun YH, Park YS, Lee ES, Bang SM, Heo DS, Park SY, et al. Validation of the Korean version of the EORTC QLQ-C30. Qual Life Res 2004;13(4):863-8.

17. Blazeby JM, Conroy T, Hammerlid E, Fayers P, Sezer O, Koller M, et al:; European Organisation for Research and Treatement of Cancer Gastrointestinal and Quality of Life Groups. Clini- cal and psychometric validation of an EORTC questionnaire module, the EORTC QLQ-OES18, to assess quality of life in patients with oesophageal cancer. Eur J Cancer 2003;39(10): 1384-94.

18. Memarian R. Application of fundamentals and theories in nursing. Tehran: University of Tarbiat Modares Press; 2004; 76-90.

19. Cella D, Eton DT, Lai JS, Peterman AH, Merkel DE. Combining anchor and distribution-based methods to derive minimal clinically important differences on the Functional Assessment of Cancer Therapy (FACT) anemia and fatigue scales. J Pain Symptom Manage 2002;24(6):547-61

20. Norkin CC, White DJ. Measurement of joint motion: a guide to goniometry. Philadelphia: F.A. Davis; 1985.

21. Butt Z, Wagner LI, Beaumont JL, Paice JA, Peterman AH, Shevrin D, et al. Use of a single-item screening tool to detect clinically significant fatigue, pain, distress, and anorexia in ambulatory cancer practice. J Pain Symptom Manage 2008; 35(1):20-30.

22. Toshkezi G, Dejesus J, Jabre JF, Hohler A, Davies K. Long thoracic neuropathy caused by an apical pulmonary tumor. J Neurosurg 2009;110(4):754-7.

23. Hamada J, Igarashi E, Akita K, Mochizuki T. A cadaveric study of the serratus anterior muscle and the long thoracic nerve. J Shoulder Elbow Surg 2008;17(5):790-4.

24. Shin AR, Lee JH, Kim DE, Cynn HS. Thera-band application changes muscle activity and kyphosis and scapular winging during knee push-up plus in subjects with scapular winging: the cross-sectional study. Medicine (Baltimore) 2018;97(14): e0348.

25. Januario LB, Madeleine P, Cid MM, Samani A, Oliveira AB. Can exposure variation be promoted in the shoulder girdle muscles by modifying work pace and inserting pauses during simulated assembly work? Appl Ergon 2018;66:151-60.

26. National Cancer Institute. Coordinating Center for Clinical Trials (CCCT). National Cancer Institute [Internet]. Rockville: 2010 [cited 2020 May 2]. Available from: http://transformingtrials.cancer.gov/steeringcommittees/symptom-management.

27. Hardwick DH, Beebe JA, McDonnell MK, Lang CE. A comparison of serratus anterior muscle activation during a wall slide exercise and other traditional exercises. J Orthop Sports Phys Ther 2006;36(12):903-10.

28. Pool MK, Nadrian H, Pasha N. Effects of a self-care educa- 
tion program on quality of life after surgery in patients with esophageal cancer. Gastroenterol Nurs 2012;35(5):332-40.

29. Anderson KO, Getto CJ, Mendoza TR, Palmer SN, Wang XS, Reyes-Gibby CC, et al. Fatigue and sleep disturbance in patients with cancer, patients with clinical depression, and community-dwelling adults. J Pain Symptom Manage 2003;25(4): 307-18.

30. Dirksen SR, Epstein DR. Efficacy of an insomnia intervention on fatigue, mood and quality of life in breast cancer survivors. J Adv Nurs 2008;61(6):664-75.

31. Mantoan S, Cavallin F, Pinto E, Saadeh LM, Alfieri R, Cagol M, et al. Long-term quality of life after esophagectomy with gastric pull-up. J Surg Oncol 2018;117(5):970-6.

32. Gannon JA, Guinan EM, Doyle SL, Beddy P, Reynolds JV, Hussey J. Reduced fitness and physical functioning are longterm sequelae after curative treatment for esophageal cancer: a matched control study. Dis Esophagus 2017;30(8):1-7.

33. Watanabe M, Okamura A, Toihata T, Yamashita K, Yuda M, Hayami M, et al. Recent progress in perioperative management of patients undergoing esophagectomy for esophageal cancer. Esophagus 2018;15(3):160-4. 